\title{
PRIMERA CONTRIBUCIÓN AL CONOCIMIENTO DE MACROMICETES DE LA VERTIENTE NORTE DEL CERRO EL CÍPIL, EN LA COSTA SUR DE JALISCO
}

\author{
Edgar Everardo Padilla-Velarde ${ }^{1}$, Georgina Zarco-Velazco ${ }^{1}$, Laura Guzmán- \\ DÁVALOS $^{2}$ Y RAMÓN CUEVAS-GUZMÁN ${ }^{1}$ \\ ${ }^{1}$ Universidad de Guadalajara, Instituto Manantlán de Ecología y Conservación de \\ la Biodiversidad, Centro Universitario de la Costa Sur, Avenida Independencia \\ Nacional 151, 48900 Autlán de Navarro, Jalisco, México. \\ ${ }^{2}$ Universidad de Guadalajara, Instituto de Botánica, Departamento de Botánica y \\ Zoología, Centro Universitario de Ciencias Biológicas y Agropecuarias, Apdo. \\ postal 1-139, 45101 Zapopan, Jalisco, México. \\ Autor para la correspondencia: rcuevas@cucsur.udg.mx
}

\section{RESUMEN}

Se realizaron 18 exploraciones a la vertiente norte del cerro El Cípil, ubicado al oeste del municipio de Casimiro Castillo, en la costa sur del estado de Jalisco, México, durante los meses de junio a octubre de 2006 y 2007. Se recolectaron 441 especímenes de hongos, de los que se determinaron 151 que corresponden con 101 especies, las cuales pertenecen a Ascomycota (12) y Basidiomycota (87) del reino Fungi y a Myxogastrea (2) del reino Protozoa. Aun cuando los resultados son preliminares, se registran 15 especies por primera vez para el estado de Jalisco, para las cuales se proporciona una breve descripción e ilustraciones de sus principales caracteres microscópicos. Se analiza la distribución de las especies por comunidades vegetales y sustrato, además de informar de su importancia para el hombre.

Palabras clave: Ascomycota, Basidiomycota, Casimiro Castillo, Myxogastrea, vegetación tropical.

\section{ABSTRACT}

Eighteen explorations were conducted from June to October of 2006 and 2007 to the northern slopes of mount El Cípil, located to the west of the municipality of Casimiro 
Castillo, on the south coast of the state of Jalisco, Mexico. In total, 441 fungal specimens were collected, of which 151 were identified, corresponding to 101 species, which belong to Ascomycota (12) and Basidiomycota (87) of the Fungi kingdom and to Myxogastrea (2) of the Protozoa kingdom. Although the results are preliminary, fifteen species are reported for the first time for the state of Jalisco, for which a brief description and illustrations of their main microscopic characters are provided. Furthermore, an analysis of the distribution of the species by plant communities and substrate, as well as information about their usefulness for man, is presented.

Key words: Ascomycota, Basidiomycota, Casimiro Castillo, Myxogastrea, tropical vegetation.

\section{INTRODUCCIÓN}

El cerro El Cípil se localiza al oeste del municipio de Casimiro Castillo, Jalisco, México (Fig. 1). Tiene una extensión superficial de 10569 ha distribuida en un gradiente altitudinal de 200 a 1550 m s.n.m., y su vertiente norte cubre una área de 3531 ha (Centro de Estudios del Territorio Nacional, 1972a, 1972b). El clima es el más húmedo de los cálidos subhúmedos (Secretaría de Programación y Presupuesto, 1981), con una estación lluviosa de finales de mayo o principios de junio a septiembre. La precipitación media anual es de $1637 \mathrm{~mm}$ y la temperatura va de los 19 a los $36^{\circ} \mathrm{C}$ con una media anual de $27^{\circ} \mathrm{C}$ (Anónimo, 2003). La zona alberga los siguientes tipos de vegetación: bosque tropical subcaducifolio ( $25 \%$ de la superficie), bosque de encino (43\%), pastizales (10\%) y áreas agrícolas (22\%) (Guzmán-Fregoso, 2013).

Los estudios sobre hongos mexicanos se han realizado principalmente en bosques de pino o encino, en tanto que en la vegetación tropical y subtropical, que concentra una gran riqueza de especies fúngicas, se han hecho menos a pesar de presentar mayor pérdida de su extensión original (Guzmán, 1995). Es por ello que las investigaciones en estos ambientes requieren de una atención especial (Guzmán, 2004). El trabajo más reciente de compilación de información sobre los hongos del estado de Jalisco registró 1040 especies, en su mayoría procedentes de nueve municipios de la entidad (Sánchez-Jácome y Guzmán-Dávalos, 2011), por lo que se hace necesario llevar a cabo más exploraciones y estudios taxonómicos de estos organismos para contar con un inventario aceptable para el estado (Anónimo, 1998).

El cerro El Cípil reviste importancia por ser un área de recarga de mantos acuíferos para parte de los municipios de Casimiro Castillo, La Huerta y 


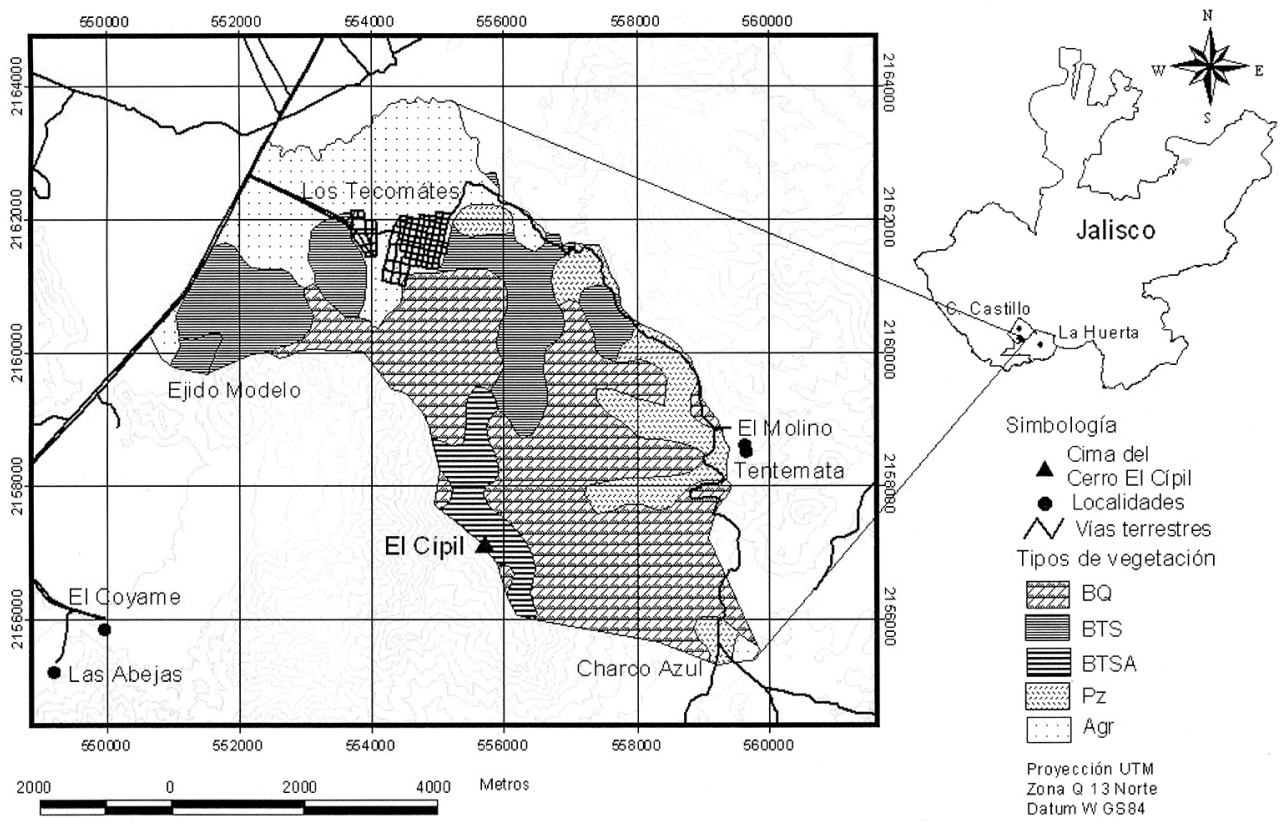

Fig. 1. Localización del cerro El Cípil y el área de estudio en el municipio de Casimiro Castillo, Jalisco, México: Agr = agricultura, BTSA = bosque tropical subcaducifolio de altura, $\mathrm{BQ}=$ bosque de Quercus, $\mathrm{BTS}=$ bosque tropical subcaducifolio, $\mathrm{Pz}=$ pastizal.

Cuautitlán (Guzmán-Fregoso, 2013), y porque al menos 25\% de su superficie está cubierta por bosque tropical subcaducifolio. Se desconoce la existencia de alguna investigación sobre los hongos en esta región del estado de Jalisco, por lo cual el trabajo busca contribuir al conocimiento micológico de esta entidad a través de un inventario preliminar de los hongos en la vertiente norte del cerro El Cípil, analizar la distribución de las especies por sustratos y tipos de vegetación, indagar sobre los usos por el hombre y describir e ilustrar las principales características microscópicas distintivas de los registros nuevos para el estado de Jalisco.

\section{MATERIALES Y MÉTODOS}

De junio a octubre de 2006 y 2007 se realizaron 18 exploraciones micológicas en el bosque tropical subcaducifolio y bosque de encino del área de 
estudio, en las que se obtuvieron 441 ejemplares. Las recolectas se hicieron cubriendo la mayor área posible y se dirigieron a todos los esporomas visibles de todos los grupos, con la finalidad de captar la mayor cantidad de especies, tal y como se recomienda para hacer un inventario micológico (Cifuentes et al., 1986). Durante los recorridos de campo se registró el tipo de vegetación y el sustrato donde se encontraron los especímenes y se elaboraron sus descripciones macromorfológicas en fresco (Guzmán, 1977; Velásquez et al., 1987), para luego ser deshidratados y depositados en la colección micológica del Herbario ZEA del Centro Universitario de la Costa Sur, con duplicados para el Herbario del Instituto de Botánica del Centro Universitario de Ciencias Biológicas y Agropecuarias (IBUG), ambos de la Universidad de Guadalajara. Se realizaron cortes de los esporomas, principalmente del himenóforo, que se montaron en $\mathrm{KOH}$ a 5\% (Largent et al., 1977), para luego observarlos al microscopio óptico. Las estructuras microscópicas de mayor importancia taxonómica se describieron a detalle y se dibujaron a escala.

La determinación de las especies se hizo atendiendo a la morfología del hongo, tanto de caracteres macro como micromorfológicos, para lo cual se utilizaron las obras de Dennis (1970), Guzmán (1977), Pegler (1983a), Breitenbach y Kränzlin (1986, 2000), Gilbertson y Ryvarden $(1986,1987)$, entre otras.

El catálogo fúngico se elaboró de acuerdo con el orden taxonómico del Index Fungorum (2012) y Mycobank (2012). Para cada espécimen se proporciona su número de recolección, tipo de vegetación, sustrato e importancia para el hombre. Las especies que constituyen registros nuevos para el estado de Jalisco se describieron de manera breve y se ilustraron las estructuras micromorfológicas de mayor valor taxonómico.

Para el análisis de la distribución de los macromicetes por comunidades vegetales y sustrato, se consideró la información observada directamente en campo a partir del registro de la vegetación y el sustrato donde se encontraron. La importancia de las especies de hongos para el hombre se obtuvo de la revisión de los trabajos de Guzmán (1994a, 2004, 2008a, b), Pérez-Moreno et al. (2008) y Shepard et al. (2008). Debido a que algunas de ellas se comparten entre diferentes tipos de vegetación, sustratos o usos para el hombre, la suma podría ir a más de 101 registros, por lo que se hizo el ajuste respectivo para llevarlo a 100\%. Los resultados y la discusión se hicieron con base en los 151 ejemplares determinados de los 441 recolectados (34.2\% del total), por lo que se trata de una interpretación preliminar para el área de estudio. 


\section{RESULTADOS Y DISCUSIÓN}

Inventario micológico

Se recolectaron 441 macromicetes en la cara norte del cerro El Cípil. Los 151 ejemplares determinados corresponden a 101 especies, 73 géneros, 44 familias y 19 órdenes. De ellos, 12 especies (11.8\%) son Ascomycota, 87 (86.1\%) Basidiomycota del reino Fungi y 2 (1.9\%) Myxogastrea del reino Protozoa. Se encontraron 15 especies (14.8\%) que representan registros nuevos para el estado de Jalisco (Cuadro 1).

Basidiomycota es el grupo más rico en especies, lo cual era de esperar, ya que típicamente forman esporomas macroscópicos, mientras que los Ascomycota, a pesar de constituir el grupo de hongos más diverso en la naturaleza, en su mayoría son microscópicos, por lo que están poco representados en los estudios de macromicetes. Ascomycota cuenta con tres órdenes en este inventario; el de mayor riqueza de especies es Xylariales con 8 (7.9\%). Los Basidiomycota están representados por 14 órdenes, de los cuales Agaricales es el más rico con 34 especies (33.6\%), le siguen los Polyporales con 24 (23.7\%), Boletales con 6 (5.9\%) y Russulales con 5 (4.9\%).

Las familias con más géneros pertenecen a Polyporaceae con 10 (13.6\%), Xylariaceae con 7 (9.5\%) y Marasmiaceae con 4 (5.4\%), lo cual es un patrón esperado si consideramos que estos grupos están muy bien representados en México (San Martín et al., 1998; Guzmán, 2004; Valenzuela et al., 2006). Los géneros más ricos en especies son Amanita (4), Inocybe (4), Russula (4), Auricularia (3), Marasmius (3), Ganoderma (3) y Hexagonia (3). Otros que destacan en el área son Agaricus, Collybia, Hygrophorus, Mycena y Lepiota, de los que sólo pocas o ninguna especie se pudieron determinar.

Los representantes de Myxogastrea se incluyen de manera tradicional en los inventarios de macromicetes, ya que forman fructificaciones de microscópicas a frecuentemente milimétricas, en ocasiones de varios centímetros (Herrera y Ulloa, 1998). Al no seguirse métodos especializados para su recolección, generalmente son subestimados en los inventarios, como ocurrió en esta investigación.

Distribución de los macromicetes por comunidades vegetales

Se determinaron 11 especies de Ascomycota para el bosque tropical subcaducifolio (BTS) y dos para el bosque de encino o Quercus (BQ). Los Basidiomycota se encuentran representados por 51 especies en el BTS y 51 en el BQ, de las cuales 16 son compartidas. De la división Myxogastrea se determinaron dos representantes, ambos del BTS. El mayor número de especies corresponde al BTS con 64, en 53 géneros, de las cuales solo 16 se comparten con el $\mathrm{BQ}$. Los géneros con más espe- 
cies en el BTS, con base en el material determinado, son Auricularia, Ganoderma y Hexagonia con tres y Clavulinopsis, Geastrum, Lentinus, Leucocoprinus, Marasmius y Xylaria con dos especies cada uno (Cuadro 1).

Se determinaron para el BQ 53 especies, incluidos en 42 géneros (Cuadro 1). Aquellos con más especies son Amanita (4), Inocybe (4), Russula (4), Auricularia (2) y Psilocybe (2), lo que coincide con las investigaciones de Fierros y GuzmánDávalos (1995), Nava y Valenzuela (1997) y Chanona-Gómez et al., (2007). Guzmán (1995) señaló a la vegetación tropical y subtropical como rica en especies fúngicas, lo cual es consistente con lo registrado en el área de estudio.

El bosque de Quercus estudiado se puede considerar que manifiesta influencia tropical, lo que es evidente por la presencia de Pycnoporus sanguineus, el cual es un indicador de zonas de clima cálido y con perturbación (Guzmán, 1994b). Chanona-Gómez et al. (2007) atribuyen una mayor riqueza de especies de hongos en la selva mediana que en el $\mathrm{BQ}$, a una descomposición más rápida de la materia orgánica, debido a las temperaturas más altas y la humedad más pronunciada.

Los esfuerzos de recolección son comparables entre el BTS y el BQ, sin embargo, el mayor número de especímenes corresponde al BTS (60\%). Si se consideran los ejemplares no determinados, pero claramente diferenciados, el BTS sigue siendo el que presenta más diversidad de taxones.

La afinidad de las especies por determinado tipo de vegetación en el área de estudio, tiene similitud con lo registrado por Fierros et al. (2000) y Chanona-Gómez et al. (2007), quienes señalaron que cada comunidad vegetal tiene una micobiota característica y generalmente se comparten pocas especies entre ellas.

Distribución de los macromicetes por sustrato

Se registran 56 (55.4\%) taxones lignícolas, 23 (22.7\%) micorrizógenos, 11 (10.9\%) humícolas, 5 (4.9\%) fimícolas, 3 (3\%) terrícolas, 2 (2\%) fungícolas y 1 (1\%) entomopatógeno. En el BTS se encuentra un número mayor de especies de hongos que crecen sobre madera, con 48 y solo 22 en el BQ. Ya se ha consignado que los últimos son más diversos en las zonas tropicales que en las templadas (Guzmán-Dávalos y Guzmán, 1979), con preferencia por áreas con cierto grado de perturbación (Guzmán, 1983). Las familias mejor representadas en este grupo son Polyporaceae con 14 especies, Xylariaceae con ocho, Ganodermataceae con cuatro, y Auriculariaceae y Marasmiaceae con tres. Los géneros con más especies son Auricularia (3), Ganoderma (3) y Hexagonia (3).

El grupo de las micorrizógenas, es el segundo más diverso, todas del BQ. Las familias mejor representadas son Amanitaceae, Inocybaceae y Russulaceae, 
con cuatro especies cada una y Boletaceae con tres. Los géneros con más especies en esta categoría corresponden a Amanita, Inocybe y Russula, con cuatro cada uno. Dentro del BTS se recolectaron esporomas de un representante de Boletaceae que no fue determinado, lo cual sugiere la presencia de ectomicorrizas en la vegetación tropical del área. Es importante resaltar la relevancia forestal que tienen tales hongos ectomicorrizógenos (Pegler, 1983a; Montiel-Arcos et al., 1984), ya que la correspondiente simbiosis representa una de las asociaciones mutualistas más prominentes y ecológicamente cruciales en los hábitats terrestres (Rinaldi et al., 2008).

Los hongos humícolas están representados por 11 especies, de las cuales diez se registran para el BTS y una para el BQ. Los géneros mejor representados son Marasmius (3 especies), Geastrum (2) y Leucocoprinus (2). Su importancia radica en que participan activamente en la degradación de la materia orgánica en el ecosistema (Herrera et al., 2006). Se registran cinco especies fimícolas, en Panaeolus (2), Psilocybe (2) y Poronia (1), de las cuales tres se encuentran en el BQ, una en el BTS y una compartida. Para el grupo de las terrícolas se consignan tres: Clavulinopsis laeticolor del BTS, Phallus indusiatus del BQ y BTS y Agaricus silvaticus del BQ. Los dos representantes de Tremella, uno en BQ y el otro en BTS, son parásitos de otros hongos. Cordyceps militaris, la única entomopatógena se encontró sobre una larva de lepidóptero en el BTS.

Importancia para el hombre

Las especies por su significación para el hombre se agruparon en las categorías de destructoras de la madera, comestibles, micorrizógenas, medicinales, venenosas, fitopatógenas y alucinógenas. Las destructoras de la madera son 29, algunas de la cuales pueden crecer sobre madera firme de cercas y construcciones, generando pérdidas económicas al causar su pudrición (Chio y Guzmán, 1982). Las comestibles son 28 (Guzmán, 2008a; Pérez-Moreno et al., 2008; Shepard et al., 2008); aunque el valor de algunas de ellas es pobre, debido a su escasa carnosidad. En el área de estudio no existe el hábito de consumir estos organismos.

Se registran 23 especies potencialmente micorrizógenas, es decir asociadas con las raíces de las plantas en una simbiosis muy útil desde el punto de vista forestal (Díaz et al., 2005).

Se encontraron en el área de estudio 15 especies que se han registrado con propiedades medicinales, que pueden ser utilizadas en el tratamiento de ciertos padecimientos en el humano o que se emplean en la medicina tradicional en Mesoamérica (Guzmán, 1994a, 2008b). El grupo de los hongos venenosos presenta 11 
taxones. Los fitopatógenos están representados por seis especies. Sólo Psilocybe cubensis es alucinógena, hongo tropical común sobre el estiércol vacuno y equino (Guzmán, 2004).

Registros nuevos para Jalisco

Se encontraron 15 registros nuevos para el estado de Jalisco, de las 101 especies determinadas para la zona norte del cerro El Cípil. Para cada una se presenta una breve descripción e ilustraciones.

1. Clavulinopsis fusiformis (Sowerby) Corner, Ann. Bot. Mem. 1: 367 (1950) Figs. $2-3$.

Basidioma de 10-18 × 0.6-0.8 mm, angostándose hacia la base, clavarioide, simple, no ramificado, con el ápice subagudo a agudo. Superficie ligera e irregularmente acanalada, de color amarillo intenso a anaranjado. Consistencia carnosa a ligeramente papirácea. Basidiosporas de 5-6 6 4.5-5 $\mu \mathrm{m}$, anchamente elípticas a subglobosas, con pared delgada, lisas, amarillentas, unigutuladas, apículo conspicuo. Basidios de 35-40 × 5.5-6.5 $\mu \mathrm{m}$, claviformes, con la base delgada y alargada, con fíbula basal, tetraspóricos, hialinos.

Hábitat. En bosque tropical subcaducifolio, gregario, sobre tierra.

Material estudiado. 500-1000 m al SE de Piedra Pesada, vertiente N del cerro El Cípil, municipio de Casimiro Castillo, 395-400 m s.n.m., agosto 12 de 2006, E. Padilla 228 (ZEA); octubre 12 de 2006, E. Padilla 397 (IBUG).

Observaciones. Los ejemplares coinciden con lo descrito por Breitenbach y Kränzlin (1986) y por Gerhardt et al. (1995). Una especie similar es C. laeticolor (Berk. \& M. A. Curtis) R. H. Petersen, la cual, de acuerdo con Breitenbach y Kränzlin (1986), presenta el ápice del basidioma redondeado y basidiosporas elipsoides de apículo muy largo. Al describir estas especies ambos autores señalaron el color amarillento en las esporas únicamente para C. laeticolor. Sin embargo, las de los ejemplares de Jalisco también presentan un ligero color amarillento. García-Sandoval et al. (2005) citaron a C. fusiformis de México, sin localidad precisa.

2. Cortinarius sanguineus (Wulfen) Fr., Epic. Syst. Mycol. (Upsaliae): 288 (1838) [1836-1838] Fig. 4. 
Píleo de $15 \mathrm{~mm}$ de diámetro, más o menos cónico a plano-convexo, fibriloso, seco, de color rojo-tinto intenso. Láminas subadheridas, juntas, rojizas. Estípite de $20 \times 4 \mathrm{~mm}$, cilíndrico, rojizo. Velo parcial aracnoide, de color rojizo oscuro. Sabor a raíz. Basidiosporas de 7-9 × 4.5-5 $\mu \mathrm{m}$, elipsoides, algunas ligeramente ovoides, con pared gruesa, verrugosas, las verrugas individuales de pequeñas a muy pequeñas, haciendo lucir la superficie rugulosa, de color castaño-rojizo. Basidios de 29-33 $\times$ 5-6 $\mu \mathrm{m}$, claviformes, rojizos, tetraspóricos. Pleuro- y queilocistidios ausentes. Fíbulas presentes.

Hábitat. En bosque de Quercus, gregario, sobre tierra.

Material estudiado. $2 \mathrm{~km}$ al SE de Piedra Pesada, vertiente $\mathrm{N}$ del cerro El Cípil, municipio de Casimiro Castillo, 817 m s.n.m., septiembre 6 de 2006, E. Padilla 316 (ZEA).

Observaciones. Una de sus características distintivas es su color rojo-tinto oscuro en todo el basidioma, lo que coincide con lo descrito por Gerhardt et al. (1995). Una especie semejante es C. cinnabarinus Fr., cuyo basidioma es de color rojo claro y tiene el píleo higrófano. Cortinarius sanguineus se ha citado de Chihuahua, Durango, Hidalgo, Morelos, Nuevo León, Oaxaca y Puebla como Dermocybe sanguinea (Bandala-Muñoz et al., 1988).

3. Hypochnella violacea Auersw. ex J. Schröt., in Cohn, Krypt.-Fl. Schlesien (Breslau) 3.1(25-32): 402 (1888) [1889]

Basidioma de hasta $180 \mathrm{~mm}$ en su parte más amplia y de 3-6 $\mathrm{mm}$ de grosor, efuso, resupinado, efuso-reflejado o formando repisas de 10-40 $\times 5-25 \times 3-6 \mathrm{~mm}$, flexibles, con la superficie pubescente a villosa, ligeramente zonada, de color grisvioláceo a blanquecino, margen abultado en repisas jóvenes, adelgazado y agudo en adultos, algo sinuoso, tomentoso a ligeramente velutino o algodonoso, gris-violáceo. Contexto delgado de (0.5-) 0.8-1 mm de espesor. Himenóforo con dientes mal definidos, de 1-3.5 $(-5) \times 0.8-1(-2) \mathrm{mm}, 7-10$ dientes por $\mathrm{cm}^{2}$, sinuosos, fusionados o no, ramificados o no, de puntas principalmente aplanadas, obtusas, de color grisvioláceo claro con tono castaño. En algunas áreas se observan exudados a manera de gotas cristalinas, de color amarillento pálido. Sistema hifal monomítico, hifas de 3.8-6 $\mu \mathrm{m}$ de diámetro, con pared gruesa, hialinas, poco ramificadas, los extremos en ocasiones con escasas granulaciones. Esporas no observadas. 
Hábitat. En bosque de Quercus, sobre tronco quemado de Quercus.

Material estudiado. $2.8 \mathrm{~km}$ al SE de Piedra Pesada, vertiente $\mathrm{N}$ del cerro El Cípil, municipio de Casimiro Castillo, 980 m s.n.m., septiembre 28 de 2006, E. Padilla 384 (ZEA).

Observaciones. El ejemplar coincide con lo descrito por García-Jiménez et al. (1998), quienes citaron este hongo de Querétaro. La especie se caracteriza por su crecimiento resupinado, su himenóforo con proyecciones irregulares a manera de dientes y su color gris-violáceo. Cabe mencionar que inmediatos al margen del basidioma se encontraron algunos cúmulos de esporas de color violáceo oscuro que contaminaron el ejemplar. Estas esporas son de (6-) 6.5-7 (-9) × 5-6.5 (-7) $\mu \mathrm{m}$, subglobosas a cortamente elipsoides, gutuladas, con el ápice redondeado a principalmente truncado, con pared ligeramente engrosada, lisas, de color castaño oscuro con tono violáceo. Casualmente las dimensiones y el color coinciden con lo descrito por García-Jiménez et al. (1998). Sin embargo, este autor señaló para las esporas una pared gruesa y no mencionó si el ápice de éstas es redondeado o truncado.

4. Hypoxylon subrutilum Starbäck, Bih. K. Svenska Vetensk. Akad. Handl., Afd. 3 27(no. 3): 10 (1901) Fig. 5

Estroma de 5-50 × 5-17 × 1.5-2 mm, aplanado-pulvinado, de consistencia carbonosa, superficie de color castaño-guinda, finamente papilada, las papilas hasta de $0.9 \mathrm{~mm}$ de diámetro. Ascosporas de (15-) 16-18 (-20.5) × 7.5-8.2 (-9) $\mu \mathrm{m}$, elipsoides, naviculares, lisas, de color castaño oscuro, con ligero tono violáceo, episporio hialino, caedizo.

Hábitat. En bosque tropical subcaducifolio, sobre el tronco sin corteza de un árbol muerto.

Material estudiado. $800 \mathrm{~m}$ al SE del Ejido Modelo, vertiente $\mathrm{N}$ del cerro El Cípil, municipio de Casimiro Castillo, 300 m s.n.m., febrero 8 de 2007, E. Padilla 403 (ZEA).

Observaciones. El ejemplar coincide con lo descrito por Martin (1969), aunque el intervalo del tamaño de las ascosporas que este autor señaló para la especie es más amplio, de 12-22.5 × 5.5-10.5 $\mu \mathrm{m}$. Especies similares son H. rubiginosum (Pers.) 

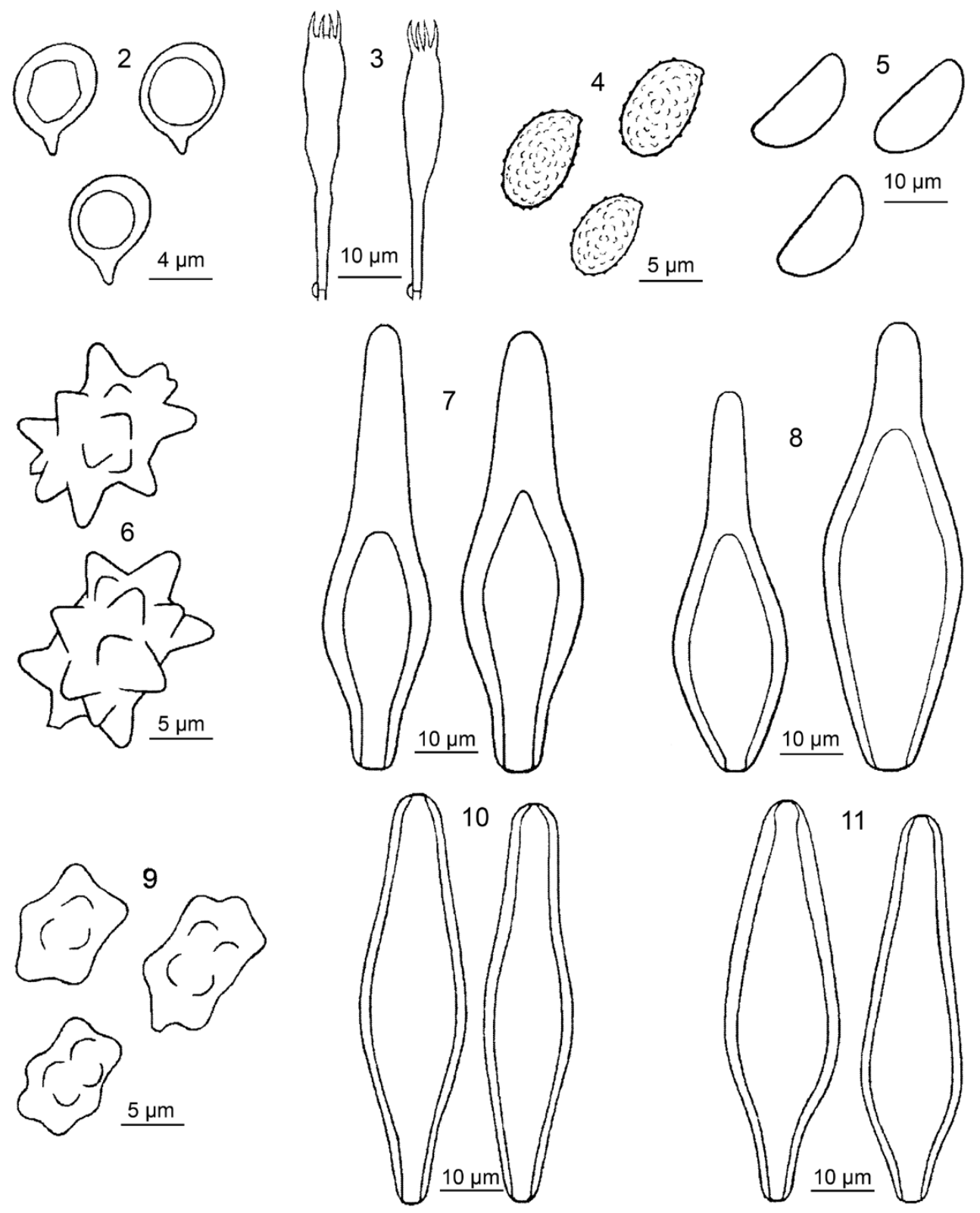

Figs. 2-11. 2-3: Clavulinopsis fusiformis, 2. basidiosporas, 3. basidios. 4: Cortinarius sanguineus, basidiosporas. 5: Hypoxylon subrutilum, ascosporas. 6-8: Inocybe calida, 6. basidiosporas, 7. pleurocistidios, 8. queilocistidios. 9-11: Inocybe lanuginosa, 9. basidiosporas, 10. pleurocistidios, 11. queilocistidios. 
Fr., que tiene ostíolos con perífisis blancas y las ascosporas de $8-14 \times 3.5-7 \mu \mathrm{m}, \mathrm{y}$ H. fuscum (Pers.) Fr., con ascosporas de 10-28 $\times$ 4.5-10.5 $\mu \mathrm{m}$, sin episporio (Martin, 1969). Se conoce de Tamaulipas y Veracruz (Medel y Chacón, 1997).

5. Inocybe calida Velen., České Houby 2: 366 (1920) Figs. 6-8

Píleo de $20 \mathrm{~mm}$ de diámetro, subcónico, superficie rimosa, con abundantes fibrillas de color castaño. Láminas subadheridas, quedando aparentemente libres, de color castaño, con ligero tono amarillento. Estípite de $50 \times 2-3 \mathrm{~mm}$, cilíndrico, de color castaño, con la superficie finamente fibrilosa, base con bulbo marginado, blanquecino. Basidiosporas de 7-10 (-11) × (5.5-) 5.8-6.7 (-7.8) $\mu \mathrm{m}$, cortamente elipsoides, con 9-13 (-14) nódulos por espora, cónicos, hasta de $3.8 \mu \mathrm{m}$ de altura, con pared gruesa o subgruesa, de color castaño-amarillento. Basidios de 25-35 $\times$ 7-9 $\mu \mathrm{m}$, claviformes, hialinos o de color castaño claro, con contenido granuloso. Pleurocistidios de 60-75 (-85) $\times(14-)$ 15-18 (-22) $\mu \mathrm{m}$, metuloides, fusoide-lageniformes, con pared gruesa, hialinos, con incrustaciones apicales cristalinas. Queilocistidios de (50-) 60-70 (-73) $\times 15-20(-23) \mu \mathrm{m}$, metuloides, lageniformes, con pared gruesa, hialinos, con incrustaciones apicales cristalinas o de color ámbar a lo largo del cuello.

Hábitat. En bosque de Quercus, sobre tierra.

Material estudiado. $1.5 \mathrm{~km}$ al SO de Piedra Pesada, vertiente $\mathrm{N}$ del cerro El Cípil, municipio de Casimiro Castillo, 391 m s.n.m., agosto 7 de 2006, E. Padilla 105 (ZEA).

Observaciones. El ejemplar coincide con lo descrito por Breitenbach y Kränzlin (2000), excepto en que las esporas presentan más nódulos y éstos son más agudos. Una especie muy relacionada es I. asterospora Quél., por su píleo radialmente rimoso y su estípite bulboso-marginado. Se distingue por sus basidiomas más grandes (píleo de 30-50 mm de diámetro, estípite de 60-70 × 3-8 $\mathrm{mm}$ ) y sus basidiosporas de 9.2-12.5 × 7.5-11 $\mu \mathrm{m}$, subglobosas, con 9-11 tubérculos muy prominentes (Breitenbach y Kränzlin, 2000). Pese a que el espécimen estudiado concuerda mejor con I. calida, el tamaño de los nódulos de las esporas es más similar al de I. asterospora. No se encontró ninguna cita de I. calida para México, por lo que probablemente corresponda con un registro nuevo para el país.

6. Inocybe lanuginosa (Bull.) P. Kumm., Führ. Pilzk. (Zerbst): 80 (1871) Figs. 9-11 
Píleo de $20 \mathrm{~mm}$ de diámetro, subcónico, superficie fibriloso-escuarrosa, de color castaño. Láminas subadheridas a casi libres, de color castaño-ocre. Estípite de $30 \times 4 \mathrm{~mm}$, cilíndrico, base bulbosa, bulbo ligeramente marginado, blanquecino a ligeramente castaño claro. Basidiosporas de (7-) 8-10 (-11.5) $\times 5-6 \mu \mathrm{m}$, elipsoides a cortamente elipsoides, con pared subgruesa, de color castaño o castaño-amarillento, con 8-10 nódulos por espora, cónico-obtusos, hasta de $1.5 \mu \mathrm{m}$ de altura. Basidios de 23-31 × 6-9 (-10) $\mu \mathrm{m}$, claviformes, con pared delgada y contenido granuloso, hialinos o de color castaño claro. Pleurocistidios de (55-) 60-70 × 12-15 (-17) $\mu \mathrm{m}$, metuloides, fusoides a fusoide-lageniformes, con pared gruesa, hialinos, con incrustaciones apicales, ápice ancho y obtuso, abundantes. Queilocistidios de (50-) 57-70 × (13-) 14-18 (-23) $\mu \mathrm{m}$, metuloides, de fusoides a fusoide-lageniformes, con pared gruesa, hialinos, con incrustaciones apicales cristalinas. Fíbulas ausentes.

Hábitat. En bosque de Quercus, sobre tierra.

Material estudiado. $1.5 \mathrm{~km}$ al SO de Piedra Pesada, vertiente $\mathrm{N}$ del cerro El Cípil, municipio de Casimiro Castillo, 391 m s.n.m., agosto 7 de 2006, E. Padilla 118 (ZEA).

Observaciones. El ejemplar coincide con lo descrito por Breitenbach y Kränzlin (2000), aunque las basidiosporas del ejemplar de Jalisco son ligeramente más angostas, ya que estos autores las señalaron de 8-10.4 × 5.5-7.3 $\mu \mathrm{m}$, aunque los mismos argumentaron que la especie suele presentar bastante variabilidad. Kong-Luz (1998) la registró de Tlaxcala, y previamente Pérez-Silva (1967) la citó de México, pero de acuerdo con Matheny y Kropp (2001) el ejemplar que estudió corresponde a otra especie.

7. Kretzschmaria deusta (Hoffm.) P.M.D. Martin, Jl. S. Afr. Bot. 36(2): 80 (1970) Fig. 12

Estroma de $100 \times 70 \times 2-3 \mathrm{~mm}$, en forma de una costra gruesa poco lobada, rugosa, fácilmente desprendible del sustrato, de color gris oscuro a blanquecino hacia el margen, el cual presenta zonaciones a manera de pliegues poco marcados, de consistencia carbonosa a correosa. Peritecios inmersos, con ostíolos papilados, negruzcos. Ascosporas de 28-33 × 8-10 $\mu \mathrm{m}$, fusoides a naviculares, con uno de sus lados plano o más o menos plano, con pared gruesa, lisas, gutuladas, de color castaño oscuro a negro. 
Hábitat. En bosque tropical subcaducifolio, sobre corteza de un árbol vivo.

Material estudiado. $1 \mathrm{~km}$ al NO de Tecomates, vertiente $\mathrm{N}$ del cerro El Cípil, municipio de Casimiro Castillo, 353 m s.n.m., agosto 3 de 2006, E. Padilla 44 (ZEA).

Observaciones. Un sinónimo de esta especie es Ustulina deusta (Hoffm.) Lind. El ejemplar coincide con lo descrito por Dennis (1981), en que se caracteriza por su desarrollo a manera de costra gruesa y arrugada sobre el tronco de los árboles y su color grisáceo-negruzco. Rodríguez et al. (1989) la registraron de Veracruz y Yucatán, causando la muerte de árboles de naranjo. Posteriormente, San Martín y Lavin (1997) la citaron de Chiapas y Tamaulipas.

8. Lentinus striatulus Lév., Ann. Sci. Nat., Bot., sér. 3 5: 120 (1846) Figs. 13-14

Píleo de 100-170 mm infundibuliforme, superficie ligeramente estriada y finamente hirsuta, blanquecina con tonos amarillentos, margen inflexo a involuto. Láminas decurrentes, blanquecinas con tonos amarillentos. Estípite de $30 \times 10 \mathrm{~mm}$, excéntrico, sinuoso, blanquecino, con pequeñas escamas adheridas, como parches, de color castaño-rojizo hacia la base. Basidiosporas de (5.2-) 6-8.5 (-9.5) × (2.2-) 2.5-3 $\mu \mathrm{m}$, cilíndricas, lisas, con pared delgada, con apículo pequeño, hialinas. Hifas generativas de (2.7-) 3-5 (-6) $\mu \mathrm{m}$, con pared delgada, hialinas, con fíbulas. Hifas esqueleto-conectivas de (2.8-) 3-6 (-11) $\mu \mathrm{m}$, con pared gruesa, poco ramificadas, hialinas. Queilocistidios no observados.

Hábitat. En bosque tropical subcaducifolio, sobre madera podrida.

Material estudiado. $500 \mathrm{~m}$ al SE de Piedra Pesada, vertiente $\mathrm{N}$ del cerro El Cípil, municipio de Casimiro Castillo, 395 m s.n.m., agosto 21 de 2006, E. Padilla 230 (ZEA).

Observaciones. El ejemplar coincide con lo descrito por Pegler (1983b). Una especie muy similar es L. scleropus (Pers.) Fr., la cual se caracteriza por presentar hifas esqueleto-conectivas muy ramificadas. Se conocía de Nayarit, Tabasco y Veracruz (Camacho, 2010).

9. Marasmius haematocephalus (Mont.) Fr., Epic. Syst. Mycol. (Upsaliae): 382 (1838) [1836-1838] Figs. 15-17 
Píleo de $12 \mathrm{~mm}$ de diámetro, convexo a campanulado, membranoso, sulcado, superficie seca y ligeramente opaca, de color rojo-vináceo. Láminas subadheridas a libres, muy separadas entre sí, blanquecinas o blanquecino-violáceas. Estípite de 50 $\times 0.5 \mathrm{~mm}$, con superficie brillante, de color castaño-rojizo oscuro, micelio basal de color crema a amarillento. Basidiosporas de (15-) 16-19 × 3.5-4 (-5) $\mu \mathrm{m}$, cilíndricolacrimoides, con uno de sus extremos aguzado, lisas, con pared delgada, hialinas. Pleurocistidios de (25-) 30-50 (-60) × (6-) 7-10 (-13) $\mu \mathrm{m}$, cilíndricos a angostamente utriformes, ligeramente sinuosos, adelgazados hacia la parte superior, a veces con constricciones hacia el ápice, subcapitados, lisos, con pared delgada, hialinos o de color pardo claro. Queilocistidios de 15-20 (-24) × 6-10 $\mu \mathrm{m}$, como células en forma de escoba, claviformes a cortamente claviformes, con el ápice muy ramificado, con pared delgada, hialina, la cual engrosa y cambia a color ámbar claro en las ramificaciones.

Hábitat. En bosque tropical subcaducifolio, sobre hojarasca en descomposición.

Material estudiado. $500 \mathrm{~m}$ al SE de Piedra Pesada, vertiente $\mathrm{N}$ del cerro El Cípil, municipio de Casimiro Castillo, 353 m s.n.m., agosto 3 de 2006, E. Padilla 52 (ZEA).

Observaciones. El ejemplar coincide con lo descrito por Pulido (1983) y por Guzmán (2004), en cuanto a basidiosporas y pleurocistidios, pero difiere en parte en los queilocistidios, ya que Guzmán (2004) los registró de 7-12 × 4-6 $\mu \mathrm{m}$. Sin embargo, coinciden con los de Pulido (1983), quien los observó de 15.3-18.4 × 5.1-8.1 $\mu$ m. Guzmán (1977) lo mencionó de México, sin localidad precisa, en bosques tropicales y subtropicales.

10. Mutinus bambusinus (Zoll.) E. Fisch., Ann. Jard. Bot. Buitenzorg 6: 30, tab. 4 \& 5, figs 26-31 (1886) Fig. 18

Huevo subgloboso a ovoide, blanquecino y ligeramente amarillento hacia la parte superior, dehiscente por una rasgadura apical, adherido al sustrato por un cordón miceliar basal blanco. Receptáculo de $25 \times 6 \mathrm{~mm}$, cónico, blanquecino, íntimamente adherido al estípite, cubierto por la gleba, la cual es ligeramente viscosa y de color castaño-oliváceo oscuro. Estípite de $70 \times 6 \mathrm{~mm}$, cilíndrico, ápice cónico, superficie finamente rugosa, de color salmón o anaranjado rosado claro, a más claro hacia la base, hueco y muy frágil. Volva membranosa, blanca o blanquecino-grisácea, con un cordón miceliar blanco en la parte inferior. Basidiosporas de (3.5-) 3.8- 
$4.6(-4.8) \times 1.5-1.8(-1.9) \mu \mathrm{m}$, elíptico-cilíndricas, con un extremo truncado, lisas, con pared delgada, sin apículo, hialinas, con tono verde pálido.

Hábitat. En bosque tropical subcaducifolio, en lugares húmedos ricos en humus.

Material estudiado. 500-1000 m al SE de Piedra Pesada, vertiente N del cerro El Cípil, municipio de Casimiro Castillo, entre los 395-425 m s.n.m., agosto 21 de 2006, E. Padilla 236 (ZEA); septiembre 12 de 2006, E. Padilla 351 (IBUG).

Observaciones. Los ejemplares coinciden con lo descrito por Dring (1964), excepto por que este autor describió a los huevos de color blanco. En cuanto a las basidiosporas indicó un tamaño ligeramente mayor, de 3.5-5.5 × 1.5-2 $\mu \mathrm{m}$. Guzmán (1977) mencionó que crece en selvas tropicales de México. Posteriormente, López et al. (1980) lo registraron de Veracruz.

11. Nothopanus hygrophanus (Mont.) Singer, Kew Bull. 23(4): 247 (1969) Fig. 19

Píleo de 10-50 mm de diámetro, flabeliforme, comúnmente de forma irregular, ligeramente estriado, superficie lisa, un poco áspera, blanquecina, a menudo manchada de color castaño o violáceo, de consistencia carnosa o subcarnosa. Láminas decurrentes, separadas, con intervenaciones bifurcadas hacia el margen, blanquecinas. Estípite de $15 \times 3 \mathrm{~mm}$, lateral, liso, blanquecino. Sabor amargo, ligeramente mentolado. Basidiosporas de (3.5-) 4-6 (-7) $\times(2-)$ 2.5-4 $\mu \mathrm{m}$, elipsoides, lacrimoides, con pared delgada, lisas, hialinas a ligeramente pardas. Basidios de 17-22 $\times 2.5-4 \mu \mathrm{m}$, estrechamente claviformes, tetraspóricos. Margen laminar fértil, pleuro- y queilocistidios ausentes. Fíbulas presentes.

Hábitat. En bosque de Quercus, sobre corteza o raíces secas.

Material estudiado. 300-1500 m al S y SO de Piedra Pesada, vertiente N del cerro El Cípil, municipio de Casimiro Castillo, entre los 360-451 m s.n.m., julio 8 de 2006, E. Padilla 13 (ZEA); agosto 7 de 2006, E. Padilla 114 (ZEA); septiembre 20 de 2006, E. Padilla 367 (IBUG).

Observaciones. Los ejemplares coinciden con lo descrito por Guzmán (2004) y Pegler (1983a), aunque las basidiosporas del ejemplar estudiado son ligeramente más grandes que las mencionadas por el último autor, quien las señaló de 3.4-5.5 × 
2.5-3.2 $\mu \mathrm{m}$. Guzmán (2004) indicó un intervalo un poco más amplio en el largo de las esporas para esta especie, de 4.5-6.5 $\mu \mathrm{m}$, aunque no mencionó el ancho. Una especie similar es N. eugrammus (Mont.) Singer, que se diferencia por presentar esporas de tamaño mayor, de 6-9 $\mu \mathrm{m}$ de longitud (Guzmán, 2004). Es común en bosques tropicales en México (Guzmán, 1977). Guzmán (2004) lo registró de Quintana Roo.

12. Phallus indusiatus Vent., Mém. Inst. Nat. Sci. Arts 1: 520 (1798) Fig. 20

Huevo subgloboso a ovoide, blanquecino con ligero tono gris pálido, dehiscente por una rasgadura apical, adherido al sustrato por un cordón miceliar basal blanco. Receptáculo de $30 \mathrm{~mm}$ de diámetro, cónico, con un poro en el ápice, superficie irregularmente alveolada, blanca, cubierta por la gleba, de color verde-oliváceo oscuro; una red blanca de hasta $8 \mathrm{~cm}$ de longitud cuelga desde el interior del receptáculo, constituida por filamentos de aproximadamente $1 \mathrm{~mm}$ de grosor, muy delicados. Estípite de $130 \times 20 \mathrm{~mm}$, cilíndrico, esponjoso, blanco, alveolado a poroso, superficie ligeramente papilada. Volva membranosa, blanquecina. Basidiosporas de (3-) 3.5-4.1 × 1.6-1.9 (-2) $\mu \mathrm{m}$, elipsoides a cilíndrico-elipsoides, lisas, con pared delgada, sin apículo, hialinas.

Hábitat. En bosque de Quercus y bosque tropical subcaducifolio, en lugares húmedos y ricos en humus.

Material estudiado. 200-1000 m al SE y SO de Piedra Pesada, vertiente N del cerro El Cípil, municipio de Casimiro Castillo, entre los 370-451 m s.n.m., agosto 7 de 2006, E. Padilla 154 (ZEA); agosto 16 de 2006, E. Padilla 193 (IBUG).

Observaciones. Los ejemplares coinciden con lo descrito por Arora (1986). Una especie parecida es $P$. duplicatus Bosc, la cual tiene un indusio corto de 2-3 $\mathrm{cm}$ de longitud, además de que éste y la volva son de un color rosado (Calonge et al., 2005). Guzmán (1977) mencionó que crece en selvas tropicales de México. Se conoce de Chiapas, Guerrero, Morelos, Oaxaca, Quintana Roo, Tabasco y Veracruz (López et al., 1980; Guzmán et al., 1990).

13. Stemonitis splendens Rostaf., Śluzowce Monogr. (Paryz): 195 (1875) [1874] Fig. 21

Esporangios de 16-25 × 0.3-0.5 mm, filiformes, cilíndricos, obtusos, ligeramente flexuosos, de color castaño oscuro con tono vináceo; gregarios, en grupos muy densos. 


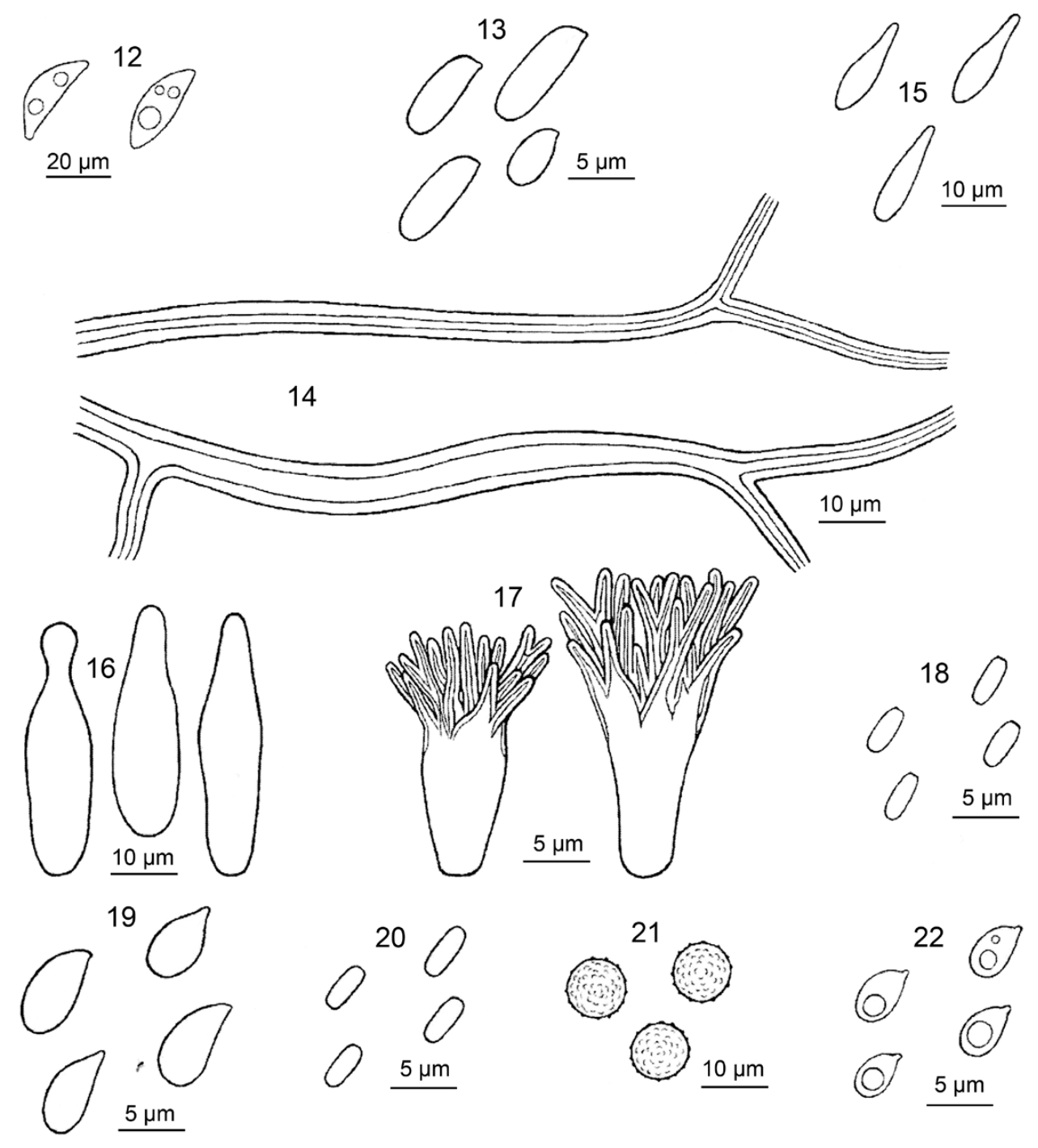

Figs. 12-22. 12: Kretzschmaria deusta, ascosporas. 13-14: Lentinus striatulus, 13. basidiosporas, 14. hifas esqueleto-conectivas. 15-17: Marasmius haematocephalus, 15. basidiosporas, 16. pleurocistidios, 17. queilocistidios; 18: Mutinus bambusinus, basidiosporas. 19: Nothopanus hygrophanus, basidiosporas. 20: Phallus indusiatus, basidiosporas. 21: Stemonitis splendens, esporas. 22: Trogia cantharelloides, basidiosporas. 
Hipotalo blanquecino-plateado. Estípite de 4-6 $\times 0.1 \mathrm{~mm}$, superficie lustrosa, de color castaño oscuro a negro. Columnela alcanzando el ápice del esporangio. Capilicio de color castaño-cobrizo a castaño oscuro, originándose de la columnela por pocas ramificaciones. Esporas de (8.7-) 9-9.5 (-10.8) $\mu \mathrm{m}$, globosas, con pared delgada a ligeramente gruesa, con la superficie diminutamente verrugosa, de color ámbar, con tono castaño a ligeramente tinto. Red del capilicio con espacios de 15-60 (-70) $\mu \mathrm{m}$ de diámetro.

Hábitat. En bosque tropical subcaducifolio, sobre tronco de árbol muerto.

Material estudiado. $4.5 \mathrm{~km}$ al $\mathrm{NO}$ de Charco Azul, vertiente $\mathrm{N}$ del cerro El Cípil, municipio de Casimiro Castillo, 990 m s.n.m., noviembre 19 de 2007, E. Padilla 439 (ZEA).

Observaciones. El ejemplar coincide con lo descrito por Martin y Alexopoulos (1969), excepto en que las esporas del material de Jalisco son un poco más grandes, ya que estos autores las describieron de 7-9 $\mu \mathrm{m}$ de diámetro; sin embargo, mencionaron que la especie es muy variable, aunque distintiva en el desarrollo típico en grupos de numerosos esporangios, densamente agrupados y con un conspicuo hipotalo blanco-plateado. Una especie afín es $S$. fusca Roth, la cual presenta esporangios más oscuros y esporas a menudo finamente reticuladas y también más oscuras (Martin y Alexopoulos, 1969). Se ha registrado de Veracruz (López et al., 1979).

14. Tremellodendron schweinitzii (Peck) G. F. Atk., J. Mycol. 8(3): 106 (1902).

Basidioma de $50 \mathrm{~mm}$ de alto, coraloide, cespitoso, de consistencia correosa, muy ramificado, con la superficie finamente lacerada y ligeramente verrugosa a lisa en algunas áreas, blanquecina en fresco con algunas máculas de color castaño claro al secarse. Ramificaciones irregularmente cilíndricas a aplanadas, ligeramente acanaladas, profusamente fusionadas, sobre todo hacia la base, blanquecinas; puntas espatuladas, aplanadas. Himenio restringido a la mitad inferior del basidioma, sobre todo hacia la base. Esporas no observadas.

Hábitat. En bosque de Quercus, sobre tierra, entre hojarasca.

Material estudiado. $600 \mathrm{~m}$ al SO de Piedra Pesada, vertiente $\mathrm{N}$ del cerro El Cípil, municipio de Casimiro Castillo, 391 m s.n.m., agosto 7 de 2006, E. Padilla 108 (ZEA). 
Observaciones. Morfológicamente el material estudiado coincide con lo descrito por Lowy (1971). Una especie similar es T. candidum (Schwein.) G. F. Atk., (ahora Dendrothele candida (Schwein.) P. A. Lemke), pero ésta presenta ramificaciones escasamente fusionadas con la mayoría de los ápices libres. Se ha registrado de Chiapas (Robles Porras et al., 2006).

15. Trogia cantharelloides (Mont.) Pat., Essai Tax. Hyménomyc. (Lons-le-Saunier): 129 (1900) Fig. 22

Píleo de 10-45 mm de diámetro, infundibuliforme, superficie glabra, de color lila-parduzco a castaño-parduzco hacia el centro. Láminas muy angostas y muy juntas entre sí, decurrentes, violáceas claras. Estípite de $30 \times 40 \mathrm{~mm}$, cilíndrico, violáceo claro a ligeramente blanquecino, con apariencia farinácea. Basidiosporas de 3.8-4.5 $\times$ 2.8-3.3 $\mu \mathrm{m}$, subelipsoides a ligeramente subglobosas, con pared delgada, lisas, con apículo evidente. Basidios de $20 \times 4-4.5 \mu \mathrm{m}$, angostamente claviformes, sinuosos, tetraspóricos. Margen fértil, sin cistidios. Fíbulas conspicuas.

Hábitat. En bosque tropical subcaducifolio, sobre madera muy podrida.

Material estudiado. 500-2500 m entre el SE y E de Piedra Pesada, vertiente N del cerro El Cípil, municipio de Casimiro Castillo, 353 m s.n.m., agosto 3 de 2006, E. Padilla 58 (ZEA); agosto 19 de 2007, E. Padilla 433 (IBUG).

Observaciones. Los ejemplares coinciden con lo descrito por Guzmán (2004), quien registró este hongo de Quintana Roo, excepto en que este autor refirió basidiomas más pequeños, de 20-30 mm de altura. Una especie afín es T. infundibuliformis Berk. \& Broome, que se diferencia por presentar basidiosporas de 6-8 $\mu \mathrm{m}$ de longitud (Guzmán, 2004).

\section{CONCLUSIONES}

La riqueza y número de registros nuevos de hongos para el estado de Jalisco, en un área tan pequeña, pone de manifiesto la necesidad de estudios micológicos en la Costa Sur de Jalisco, con el fin de poder evaluar su diversidad y así obtener un conocimiento más preciso sobre este grupo, lo cual es fundamental para establecer estrategias de uso, manejo y conservación. 


\section{LITERATURA CITADA}

Anónimo. 1998. Ordenamiento ecológico territorial del estado de Jalisco: grupo flora (hongos). Universidad de Guadalajara, Gobierno del Estado de Jalisco, Instituto Nacional de Ecología, Secretaría de Medio Ambiente, Recursos Naturales y Pesca. Zapopan, Jalisco, México. 52 pp. Documento en disco compacto.

Arora, D. 1986. Mushrooms demystified. Ed. Ten Speed Press. Berkeley, E.U.A. 957 pp.

Bandala-Muñoz, V., G. Guzmán y L. Montoya-Bello. 1988. Especies de macromicetos citadas de México, VII. Agaricales, parte II (1972-1998). Rev. Mex. Mic. 4: 205250.

Breitenbach, J. y F. Kränzlin. 1986. Fungi of Switzerland. Vol. 2. Mykologia. Lucerna, Suiza. 412 pp.

Breitenbach, J. y F. Kränzlin. 2000. Fungi of Switzerland. Vol. 5. Mykologia. Lucerna, Suiza. 338 pp.

Calonge, F. D., M. Mata y J. Carranza. 2005. Contribución al catálogo de los Gasteromycetes (Basidiomycotina, Fungi) de Costa Rica. Anales Jard. Bot. Madrid. 62(1): 23-45.

Camacho, M. 2010. Estudio taxonómico del complejo de Pleurotus, Lentinus y Panus en México. Tesis de maestría. Instituto de Ecología, A.C., Xalapa, México. pp. 16-17.

Centro de Estudios del Territorio Nacional. 1972a. Cartas temáticas (topografía, edafología y geología) de Casimiro Castillo, Jalisco. Escala 1:50 000. Clave E-13-B-22. Centro de Estudios del Territorio Nacional. México, D.F., México.

Centro de Estudios del Territorio Nacional. 1972b. Cartas temáticas (topografía, edafología y geología) de La Huerta, Jalisco. Escala 1:50 000. Clave E-13-B-32. Centro de Estudios del Territorio Nacional. México, D.F., México.

Chanona-Gómez, F., R. Andrade-Gallegos, J. Castellanos-Albores y J. Sánchez. 2007. Macromicetos del Parque Educativo Laguna Bélgica, municipio de Ocozocoautla de Espinosa, Chiapas, México. Rev. Mex. Biodiv. 78: 369-381.

Chio, R. y G. Guzmán. 1982. Los hongos de la península de Yucatán I. Las especies de macromicetos conocidas. Biotica 7(3): 385-399.

Cifuentes, J., M. Villegas y L. Pérez-Ramírez. 1986. Hongos. In: Lot, A. y F. Chiang (comp.). Manual de herbario. Consejo Nacional de la Flora de México, A.C. México, D.F., México. pp. 55-64.

Dennis, R. 1970. Fungus flora of Venezuela and adjacent countries. Kew Bull. Addit. Ser. 3: $1-531$.

Dennis, R. 1981. British Ascomycetes. Ed. J. Cramer. Vaduz, Lichtenstein. 585 pp.

Díaz, M., J. Marmolejo y R. Valenzuela. 2005. Flora micológica de bosques de pino y pinoencino en Durango, México. Ciencia UANL 8(3): 362-369.

Dring, D. 1964. Gasteromycetes of west tropical Africa. Mycol. Pap. 98: 1-60.

Fierros, M. y L. Guzmán-Dávalos. 1995. Inventario preliminar de los hongos macroscópicos de la sierra de Quila, Jalisco, México. Bol. IBUG 3(1-3): 129-142.

Fierros, M., J. Navarrete-Heredia y L. Guzmán-Dávalos. 2000. Hongos macroscópicos de la Sierra de Quila, Jalisco, México: diversidad y similitud fungística. Rev. Biol. Trop. 48(4): 931-937. 
García-Jiménez, J., D. Pedraza Kamino, C. I. Silva Barrón, R. L. Andrade Melchor y J. Castillo Tovar. 1998. Hongos del estado de Querétaro. Universidad Autónoma de Querétaro. Santiago de Querétaro, México. 199 pp.

García-Sandoval, R., E. De Luna, A. Estrada-Torres y M. Villegas, 2005. A phylogeny of Ramariopsis and allied taxa. Mycotaxon 94: 265-292.

Gerhardt, E., J. Vila y X. Llimona. 1995. Hongos de España y de Europa. Ed. Omega. Barcelona, España. 957 pp.

Gilbertson, R. y L. Ryvarden. 1986. North American polypores, 1. Abortiporus-Lindtneria. Ed. Fungiflora. Oslo, Noruega. 443 pp.

Gilbertson, R. y L. Ryvarden. 1987. North American polypores, 2. MegasporoporiaWrightoporia. Ed. Fungiflora. Oslo, Noruega. pp. 437-885

Guzmán-Fregoso, F. G. 2013. Flora arbórea del cerro El Cípil en la costa Sur de Jalisco. Tesis profesional. Centro Universitario de la Costa Sur, Universidad de Guadalajara. Autlán de Navarro, México. 47 pp.

Guzmán, G. 1977. Identificación de los hongos. Comestibles, venenosos, alucinantes y destructores de la madera. Ed. Limusa. México, D.F., México. 452 pp.

Guzmán, G. 1983. Los hongos de la Península de Yucatán. II. Nuevas exploraciones y adiciones micológicas. Biotica 8(1): 71-100.

Guzmán, G. 1994a. Los hongos en la medicina tradicional de Mesoamérica y de México. Rev. Iberoam. Micol. 11: 81-85.

Guzmán, G. 1994b. Algunos aspectos importantes en la ecología de los hongos (en especial de lo macromicetos). Ecológica 3: 1-9.

Guzmán, G. 1995. La diversidad de hongos en México. Ciencias 39: 52-57.

Guzmán, G. 2004. Los hongos de El Edén Quintana Roo (Introducción a la micobiota tropical de México). Instituto de Ecología, A.C. y Comisión Nacional para el Conocimiento y Uso de la Biodiversidad. Xalapa, México. 316 pp.

Guzmán, G. 2008a. Hongos de parques y jardines y sus relaciones con la gente. Secretaría de Educación de Veracruz. Xalapa, México. 242 pp.

Guzmán, G. 2008b. Diversity and use of traditional Mexican medicinal fungi. A review. Int. J. Med. Mush. 10: 209-217.

Guzmán, G., L. Montoya y V. M. Bandala. 1990. Las especies y formas de Dictyophora (Fungi, Basidiomycetes, Phallales) en México y observaciones sobre su distribución en América Latina. Acta Bot. Mex. 9: 1-11.

Guzmán-Dávalos, L. y G. Guzmán. 1979. Estudio ecológico comparativo entre los hongos (macromicetos) de los bosques tropicales y los de coníferas del sureste de México. Bol. Soc. Mex. Mic. 13: 89-125.

Herrera, T. y M. Ulloa. 1998. El reino de los hongos. Micología básica y aplicada. Ed. Fondo de Cultura Económica y Universidad Nacional Autónoma de México. México, D.F., México. 552 pp.

Herrera, T., E. Pérez-Silva y H. Valenzuela. 2006. Nueva contribución al conocimiento de los macromicetos de la Reserva Ecológica del Pedregal San Ángel, D.F., México. Rev. Mex. Biodiv. 77: 51-57.

Index Fungorum. 2012. Disponible en: http://www.indexfungorum.org/names/names.asp. Consultado en mayo de 2012. 
INIFAP. 2003. Estadística climatológica básica del estado de Jalisco (Periodo 1961-2000). Libro Técnico Núm. 1. Instituto Nacional de Investigaciones Forestales, Agrícolas y Pecuarias. Ed. Conexión Grafica. Guadalajara, México. 281 pp.

Kong-Luz, A. 1998. Ectomycorrhizal Agaricales of Tlaxcala, Mexico. McIlvainea 13(2): 13-24.

Largent, D., D. Johnson y R. Watling. 1977. How to identify mushrooms to genus. III. Microscopic features. Mad River Press. Eureka, E.U.A. 148 pp.

López, A., D. Martínez y J. García. 1980. Phallales conocidos del Estado de Veracruz. Bol. Soc. Mex. Mic. 14: 39-49.

López, A., A. Sosa y L. Villarreal. 1979. Estudio sobre los Myxomycetes del Estado de Veracruz, I. Bol. Soc. Mex. Mic. 13: 127-144.

Lowy, B. 1971. Tremellales. Flora Neotropica. Monograph No. 6. New York Botanical Garden. Hafner Publishing Company. Nueva York, E.U.A. 153 pp.

Martin, P. 1969. Studies in the Xylariaceae: V. Euhypoxylon. S. Afr. J. Bot. 35(3): 149-206.

Martin, G. y C. Alexopoulos. 1969. The Myxomycetes. Ed. University of Iowa Press. Iowa, E.U.A. 496 pp.

Matheny, P. B. y B. R. Kropp. 2001. A revision of the Inocybe lanuginosa group and allied species in North America. Sydowia 53(1): 93-139.

Medel, R. y S. Chacón. 1997. Ascomycetes poco conocidos de México VIII. Algunas especies del bosque mesofilo de Veracruz. Acta Bot. Mex. 39: 43-52.

Montiel-Arcos E., L. López y G. Guzmán. 1984. El género Amanita en el estado de Morelos. Biotica 9: 223-242.

Mycobank. 2012. Disponible en: www.mycobank.org/MycoTaxo.aspx. Consultado en mayo de 2012.

Nava, I. R y R. Valenzuela. 1997. Los macromicetos de la Sierra de Nanchititla. Polibotánica 5: 21-36.

Pegler, D. 1983a. Agaric flora of the Lesser Antilles. Ed. Her Majesty's Stationery Office. Londres, Inglaterra. $668 \mathrm{pp}$.

Pegler, D. 1983b. The genus Lentinus. A world monograph. Ed. Her Majesty's Stationery Office. Londres, Inglaterra. $281 \mathrm{pp}$.

Pérez-Moreno, J., M. Martínez-Reyes, A. Yescas-Pérez, A. Delgado-Alvarado y B. Xoconostle-Cázares. 2008. Wild mushroom markets in central Mexico and a case study at Ozumba. Econ. Bot. 62: 425-436.

Pérez-Silva, E. 1967. Les Inocybes du Mexique. An. Inst. Biol. Univ. Nal. Aut. Méx., Ser. Bot. 38(1): 1-60.

Pulido, M. 1983. Estudios en agaricales colombianos. Los hongos de Colombia IX. Instituto de Ciencias Naturales, Museo de Historia Natural, Biblioteca J. J. Triana 7, Universidad Nacional de Colombia. Bogotá, Colombia. 143 pp.

Rinaldi, A., O. Comandini y T. Kuyper. 2008. Ectomycorrhizal fungal diversity: separating the wheat from the chaff. Fungal Divers. 33: 1-45.

Robles Porras, L., M. Ishiki Ishihara y R. Valenzuela. 2006. Inventario preliminar de los macromicetos en los Altos de Chiapas, México. Polibotánica 21: 89-101.

Rodríguez, R. J. F., S. Osada Kawasoe y S. E. Cárdenas. 1989. Estudios sobre un xylariaceo relacionado con la muerte de árboles de cítricos. Agrociencia 77: 103-122. 
San Martín, F. y P. A. Lavin. 1997. Datos sobre los géneros Entonaema y Ustulina (Pyrenomycetes, Xylariaceae). Acta Bot. Mex. 40: 25-35.

San Martín, F., J. Rogers y Y.-M. Ju. 1998. Clave dicotómica provisional para los géneros de la familia Xylariaceae (Pyrenomycetes, Sphaeriales) de México. Acta Bot. Mex. 42: 35-41.

Sánchez-Jácome, M. R. y L. Guzmán-Dávalos. 2011. Hongos citados para Jalisco, II. Ibugana 16: 25-60.

Secretaría de Programación y Presupuesto. 1981. Síntesis geográfica del estado de Jalisco. Coordinación General de los Servicios Nacionales de Estadística, Geografía e Informática, Secretaría de Programación y Presupuesto. México, D.F., México.

Shepard Jr., G. H., D. Arora y A. Lampman. 2008. The grace of the flood: classification and use of wild mushrooms among the highland maya of Chiapas. Econ. Bot. 62: 437-470.

Valenzuela R., M. Palacios-Pacheco, T. Raymundo y S. Bautista-Hernández. 2006. Especies de poliporáceos poco conocidas de México. Rev. Mex. Biodiv. 77: 35-49.

Velásquez, L., Y. Saldarriaga, G. García y F. Pineda. 1987. Técnicas para la recolección y estudio macroscópico de hongos macromicetos. Acta Biol. Col. 16(60): 47-52.

Recibido en octubre de 2012.

Reactivado en diciembre de 2013.

Aceptado en octubre de 2015. 
Cuadro 1. Especies estudiadas de la vertiente norte del cerro El Cípil. BQ = bosque de Quercus; $\mathrm{BTS}=$ bosque tropical subcaducifolio; $\mathrm{E}=$ Entomófilo; $\mathrm{F}=$ Fimícola; $\mathrm{Fu}=$ Fungícola; $\mathrm{H}=$ Humícola; $\mathrm{L}=$ Lignícola; $\mathrm{T}=$ Terrícola; $\mathrm{A}=$ Alucinógeno; $\mathrm{C}=$ Comestible; $\mathrm{DM}=$ Destructor de madera; $\mathrm{M}=$ Micorrizógeno; $\mathrm{Me}=$ Medicinal; $\mathrm{PI}=$ Parásito de insectos; $\mathrm{PP}=$ Parásito de plantas; $\mathrm{V}=$ Venenoso; * = registro nuevo para el estado de Jalisco; EP = Edgar Padilla.

Especie

Vegetación Gremio Importancia Colector y Núm.

\section{FUNGI}

ASCOMYCOTA

SORDARIOMYCETES

Hypocreales

Clavicipitaceae

Cordyceps militaris (L.) Link

$\begin{array}{llll}\text { BTS } & \text { PI }\end{array}$

Xylariales

Xylariaceae

Daldinia concentrica (Bolton) Ces. \& De Not.

BQ/BTS L DM/Me EP 4, 112, 207

Entonaema pallida G.W. Martin.

BTS

L

EP 163

*Hypoxylon subrutilum Starbäck

BTS

$\mathrm{L}$

EP 403

* Kretzschmaria deusta (Hoffm.) P.M.D.

BTS

$\mathrm{L}$

EP 44 Martin

Phylacia poculiformis (Kunze) Mont.

BTS L

EP 283

Poronia oedipus (Mont.) Mont.

BQ

F

EP 421

Xylaria hypoxylon (L.) Grev.

BTS

EP 29

Xylaria polymorpha (Pers.) Grev.

BTS

$\mathrm{L}$

EP 192, 206, 284

\section{PEZIZOMYCETES}

Pezizales

Pyronemataceae

Scutellinia scutellata (L.) Lambotte

BTS

$\mathrm{H}$

EP 391

Sarcoscyphaceae

Cookeina tricholoma (Mont.) Kuntze

BTS

$\mathrm{L}$

EP 48, 398

Phillipsia domingensis Berk

BTS

$\mathrm{L}$

EP 189, 231

\section{BASIDIOMYCOTA}

AGARICOMYCETES

Agaricales

Agaricaceae 
Cuadro 1. Continuación.

\begin{tabular}{|c|c|c|c|c|}
\hline Especie & Vegetación & Gremio & Importancia & $\begin{array}{l}\text { Colector y } \\
\text { Núm. }\end{array}$ \\
\hline Agaricus sylvaticus Schaeff. & BQ & $\mathrm{T}$ & $\mathrm{C}$ & EP 90 \\
\hline Leucocoprinus birnbaumii (Corda) Singer & BTS & $\mathrm{H}$ & & EP 335 \\
\hline $\begin{array}{l}\text { Leucocoprinus cepistipes (Sowerby) Pat. } \\
\text { Amanitaceae }\end{array}$ & BTS & $\mathrm{H}$ & & EP 349 \\
\hline Amanita gemmata (Fr.) Bertill. & BQ & M & M & $\begin{array}{l}\text { EP } 81,87, \\
\quad 301\end{array}$ \\
\hline Amanita magnivelaris Peck & BQ & M & M & EP 132 \\
\hline Amanita straminea Lam. & BQ & M & M & EP 129 \\
\hline $\begin{array}{l}\text { Amanita vaginata (Bull.) Lam. } \\
\text { Clavariaceae }\end{array}$ & BQ & M & $\mathrm{C} / \mathrm{M}$ & EP 102,438 \\
\hline Clavulinopsis corniculata (Schaeff.) Corner & BTS & $\mathrm{H}$ & & EP 165 \\
\hline $\begin{array}{l}\text { *Clavulinopsis fusiformis (Sowerby) } \\
\text { Corner }\end{array}$ & BTS & $\mathrm{T}$ & & EP 228, 397 \\
\hline Cortinariaceae & & & & \\
\hline $\begin{array}{l}\text { *Cortinarius sanguineus (Wulfen) Fr. } \\
\text { Fistulinaceae }\end{array}$ & BQ & M & $\mathrm{M} / \mathrm{V}$ & EP 316 \\
\hline $\begin{array}{l}\text { Pseudofistulina radicata (Schwein.) Burds. } \\
\text { Inocybaceae }\end{array}$ & BTS & $\mathrm{L}$ & PP & EP 39 \\
\hline *Inocybe calida Velen. & BQ & M & $\mathrm{M} / \mathrm{V}$ & EP 105 \\
\hline $\begin{array}{l}\text { Inocybe geophylla (Sowerby) P. Kumm. } \\
\text { var. geophylla }\end{array}$ & BQ & M & $\mathrm{M} / \mathrm{V}$ & EP 122 \\
\hline *Inocybe lanuginosa (Bull.) P. Kumm. & BQ & M & $\mathrm{M} / \mathrm{V}$ & EP 118 \\
\hline $\begin{array}{l}\text { Inocybe rimosa (Bull.) P. Kumm. } \\
\text { Lyophyllaceae }\end{array}$ & BQ & M & $\mathrm{M} / \mathrm{V}$ & EP 69 \\
\hline $\begin{array}{l}\text { Lyophyllum decastes (Fr.) Singer } \\
\text { Marasmiaceae }\end{array}$ & BQ & M & $\mathrm{C} / \mathrm{M}$ & EP 308, 309 \\
\hline *Marasmius haematocephalus (Mont.) Fr. & BTS & $\mathrm{H}$ & & EP 52 \\
\hline Marasmius guzmanianus Singer & BTS & $\mathrm{H}$ & & EP 251 \\
\hline Marasmius oreades (Bolton) Fr. & BQ & $\mathrm{H}$ & $\mathrm{C}$ & EP 319 \\
\hline *Nothopanus hygrophanus (Mont.) Singer & BQ & $\mathrm{L}$ & $\mathrm{C}$ & $\begin{array}{l}\text { EP } 13,104, \\
367\end{array}$ \\
\hline Oudemansiella canarii (Jungh.) Höhn. & BTS & $\mathrm{L}$ & $\mathrm{C}$ & $\begin{array}{l}\text { EP } 157 \\
179,332\end{array}$ \\
\hline
\end{tabular}


Cuadro 1. Continuación.

Especie Vegetación Gremio Importancia Colector y

Núm.

*Trogia cantharelloides (Mont.) Pat.

BTS

EP 58, 433

Mycenaceae

Panellus pusillus (Pers. ex Lév.) Burds. \&

BTS

L

EP 253

O.K. Mill.

Nidulariaceae

Cyathus striatus (Huds.) Willd.

BTS

$\mathrm{L}$

EP 60

Pleurotaceae

Pleurotus djamor (Rumph. ex Fr.) Boedijn

BQ/BTS L $\quad$ C/Me EP 73, 174, 176

Pluteaceae

Pluteus cervinus (Schaeff.) P. Kumm.

BQ

L

C

EP 312

Volvariella bombycina (Schaeff.) Singer

BQ

$\mathrm{L}$

C

EP 152

Psathyrellaceae

Panaeolus antillarum (Fr.) Dennis

BQ F

V

EP 352

Panaeolus sphinctrinus (Fr.) Quél.

BTS

F

V

EP 431

Schizophyllaceae

Schizophyllum commune Fr.

BQ/BTS L C/DM/Me EP 1, 34, 70

Strophariaceae

Gymnopilus tuxtlensis Guzm.-Dáv.

BTS L

EP 226

Gymnopilus subearlei R. Valenz., Guzmán

BQ

$\mathrm{L}$

EP 298

\& J. Castillo

Psilocybe coprophila (Bull.) P. Kumm.

BQ F

F

V

EP 15, 78

Psilocybe cubensis (Earle) Singer

BQ/BTS

F

$\mathrm{A} / \mathrm{Me}$

EP 75, 432

Atheliales

Atheliaceae

*Hypochnella violacea Auersw. ex J.

BQ

L

EP 384 Schröt.

Auriculariales

Auriculariaceae

Auricularia cornea Ehrenb.

BQ/BTS

L

C/ DM /Me EP 32, 100,

187, 274,

276,429

Auricularia delicata (Mont.) Henn.

BTS

$\mathrm{L} \quad \mathrm{C} / \mathrm{DM} / \mathrm{Me} \quad \mathrm{EP} 261,374$ 
Cuadro 1. Continuación.

\begin{tabular}{lllcc}
\hline Especie & Vegetación & Gremio & $\begin{array}{c}\text { Importancia } \\
\text { Colector y } \\
\text { Núm. }\end{array}$ \\
\hline Auricularia mesenterica (Dicks.) Pers. & BQ/BTS & L & C/DM & EP 130, \\
& & & & 273,330, \\
& & & & 393
\end{tabular}

Boletales

Boletaceae

Boletellus ananas (M.A. Curtis) Murrill

BQ

M

$\mathrm{C} / \mathrm{M}$

EP 400

Strobilomyces strobilaceus (Scop.) Berk.

BQ

$\mathrm{C} / \mathrm{M}$

EP 93

Tylopilus balloui (Peck) Singer

BQ

$\mathrm{M}$

$\mathrm{M}$

EP 83, 304, 322

Diplocystidiaceae

Astraeus hygrometricus (Pers.) Morgan

BQ

M

M

EP 144

Sclerodermataceae

Scleroderma bovista Fr.

BQ

$\mathrm{M}$

$\mathrm{M} / \mathrm{V}$

EP 17, 76

Pisolithus arhizus (Scop.) Rauschert

BQ

$\mathrm{M}$

$\mathrm{M} / \mathrm{V}$

EP 77

Cantharellales

Cantharellaceae

Cantharellus cibarius Fr.

BQ

M

$\mathrm{C} / \mathrm{M}$

EP 82, 126

Clavulinaceae

Clavulina cinerea (Bull.) J. Schröt.

BTS

$\mathrm{H}$

$\mathrm{C} / \mathrm{Me}$

EP 281

Clavulina rugosa (Bull.) J. Schröt.

BQ

M

$\mathrm{C} / \mathrm{M}$

EP 120, 310

Geastrales

Geastraceae

Geastrum saccatum Fr.

BTS

$\mathrm{H}$

$\mathrm{Me}$

EP 148, 264

Geastrum triplex Jungh.

BTS

$\mathrm{H}$

$\mathrm{Me}$

EP 325

Gloeophyllales

Gloeophyllaceae

Gloeophyllum striatum (Sw.) Murrill

BTS

L

DM

EP 45

Hymenochaetales

Hymenochaetaceae

Phellinus gilvus (Schwein.) Pat.

BQ

L

$\mathrm{DM} / \mathrm{PP}$

EP 138, 435

Phallales

Phallaceae

*Mutinus bambusinus (Zoll.) E. Fisch.

BTS

H

EP 236, 351 
Cuadro 1. Continuación.

Especie Vegetación Gremio Importancia Colector y

Núm.

*Phallus indusiatus Vent.

$\begin{array}{llll}\text { BQ/BTS } & \text { T } & \text { Me } 154,193\end{array}$

Polyporales

Fomitopsidaceae

Daedalea quercina (L.) Pers.

BTS L DM EP 440

Fomitopsis feei (Fr.) Kreisel

BTS

L DM

EP 201

Ganodermataceae

Ganoderma curtisii (Berk.) Murrill.

BQ/BTS L Me/DM/PP EP 19, 68,

210,242

Ganoderma lobatum (Schwein.) G.F. Atk.

BTS L DM/PP EP 38, 47

Ganoderma resinaceum Boud.

BTS

L DM/PP

EP 282

Humphreya coffeata (Berk.) Steyaert

BTS

L

EP 65

Meripilaceae

Hydnopolyporus palmatus (Hook.) O.

BTS

L

C/PP

EP 240 Hidalgo

Rigidoporus lineatus (Pers.) Ryvarden

BQ/BTS

$\mathrm{L}$

DM

EP 137, 190

Phanerochaetaceae

Porostereum crassum (Lév.) H. Hjortstan

BTS

L

$\mathrm{DM}$

EP 386 \& Ryvarden

Meruliaceae

Byssomerulius incarnatus (Schwein.) Gilb. BQ/BTS

$\mathrm{L}$

$\mathrm{DM}$

EP 422, 424

Polyporaceae

Coriolopsis floccosa (Jungh.) Ryvarden

BTS

L

$\mathrm{DM}$

EP 407

Dichomitus mexicanus (Ryvarden)

BQ

L

DM

EP 423 Ryvarden

Favolus tenuiculus P. Beauv.

Funalia polyzona (Pers.) Niemelä

BTS

L

C

EP 31, 43

Hexagonia hydnoides (Sw.) M. Fidalgo.

BTS

$\mathrm{L}$

DM

EP 63

Hexagonia tenuis (Hook.) Fr.

BTS

L

$\mathrm{DM} / \mathrm{Me}$

EP 404

BTS

L

DM

EP 164,

338,387

Hexagonia variegata Berk.

BQ/BTS

L

$\mathrm{DM}$

EP 27, 381

Lentinus crinitus (L.) Fr.

$\mathrm{BQ} / \mathrm{BTS}$

$\mathrm{L}$

$\mathrm{C} / \mathrm{Me}$

EP 98, 103

*Lentinus striatulus Lév.

BTS

L

C

EP 230 
Cuadro 1. Continuación.

\begin{tabular}{ccc}
\hline Especie & Vegetación Gremio Importancia $\begin{array}{c}\text { Colector y } \\
\text { Núm. }\end{array}$
\end{tabular}

Lenzites elegans (Spreng.) Pat.

BTS

L

$\mathrm{DM}$

EP 21, 124, 289,347 , 385

Polyporus tricholoma Mont.

Trichaptum biforme (Fr.) Ryvarden

Trichaptum perrottetii (Lév.) Ryvarden

Pycnoporus sanguineus (L.) Murrill

Russulales

Russulaceae

Russula brevipes Peck

BQ M

$\mathrm{C} / \mathrm{M}$

EP 80

Russula cyanoxantha (Schaeff.) Fr.

BQ

$\mathrm{M}$

$\mathrm{C} / \mathrm{M}$

EP 437

Russula foetens Pers.

BQ

$\mathrm{M}$

$\mathrm{M} / \mathrm{V}$

EP 359

Russula rosea Pers.

BQ

M

$\mathrm{C} / \mathrm{M}$

EP 110, 305

Stereaceae

Stereum ostrea (Blume \& T. Nees) Fr.

BQ/BTS

L

DM

EP 5, 57, 85

Sebacinales

Sebacinaceae

*Tremellodendron schweinitzii (Peck) G.F. Atk.

BQ

M

$\mathrm{C} / \mathrm{M} / \mathrm{Me}$

EP 108

\section{DACRYMYCETES}

Dacrymycetales

Dacrymycetaceae

Dacryopinax spathularia (Schwein.) G.W. Martin

BQ/BTS

L

DM EP 28, 41, 151

\section{TREMELLOMYCETES}

Tremellales

Exidiaceae

Pseudohydnum gelatinosum (Scop.) P.

BTS

L

EP 211

Karst.

Tremellaceae

Tremella fuciformis Berk.

BQ

$\mathrm{Fu}$

C

EP 415

Tremella mesenterica Retz.

BTS

$\mathrm{Fu}$

C

EP 399 
Cuadro 1. Continuación.

\begin{tabular}{ccc}
\hline Especie & Vegetación Gremio Importancia Colector y \\
Núm.
\end{tabular}

PROTOZOA

AMOEBOZOA

MYXOGASTREA

Trichiida

Trichiaceae

Hemitrichia serpula (Scop.) Rostaf.

BTS

L

EP 213

Stemonitida

Stemonitidaceae

*Stemonitis splendens Rostaf.

BTS

$\mathrm{L}$

EP 439 